\title{
Validation of the Fujirebio Lumipulse G1200 aldosterone assay for measurements during adrenal venous sampling for primary hyperaldosteronism
}

https://doi.org/10.1515/cclm-2021-0102

Received December 16, 2020; accepted March 26, 2021;

published online April 8, 2021

Keywords: adrenal vein sampling; aldosterone; primary hyperaldosteronism.

To the Editor,

Primary aldosteronism (PA) is a group of disorders in which aldosterone production is inappropriately high for sodium status, relatively autonomous of the major regulators of secretion and non-suppressible by sodium loading [1]. PA is commonly caused by an adrenal adenoma, uni- or bi-lateral hyperplasia or in rare cases by adrenal carcinoma or familial hyperaldosteronism like glucocorticoid-remediable aldosteronism. PA results in hypertension, cardiovascular damage, sodium retention, suppression of plasma renin and increased potassium excretion [2]. Case detection of PA is performed by determining the aldosterone-renin ratio, and if divergent from normal followed by a confirmatory test (e.g. saline infusion) and ultimately CT-scan and/or adrenal venous sampling [1, 3]. Adrenal vein sampling (AVS) is used to determine whether patients suffer from primary hyperaldosteronism caused by a unilateral aldosteroneproducing adenoma or bilateral adrenal hyperplasia. The ratio between aldosterone and cortisol is used to determine whether there is lateralization and/or suppression of the adrenal glands [4]. A unilateral adenoma

\footnotetext{
*Corresponding author: Febe C. Vermue, Department of Internal Medicine, Erasmus Medical Center, Rotterdam, The Netherlands, E-mail: febevermue@gmail.com

Sjoerd A. A. van den Berg, Department of Internal Medicine, Erasmus Medical Center, Rotterdam, The Netherlands; and Department of Clinical Chemistry, Erasmus Medical Center, Rotterdam, The Netherlands
}

can be treated with laparoscopic adrenalectomy. In case of bilateral hyperplasia or when the patient is unable or unwilling to undergo surgery, it is recommended for the patient to undergo medical treatment including a mineralocorticoid receptor antagonist like spironolactone [1]. Therefore, it is adamant to assess whether aldosterone is produced uni-, or bi-lateral.

Here, we describe a novel fully automated two-step immunoassay EIA (Fujirebio Lumipulse $G$ System [Fujirebio, Inc., Tokyo, Japan]). This cartridge based automated immunoassay has a time-to-first result of $28 \mathrm{~min}$, and in contrast to LC-MS/MS can be implemented in all routine laboratories. The Lumipulse $G$ Aldosterone assay was also traceable to LC-MS/MS. Imprecision of the Fujirebio Lumipulse was CV 2.1\% (mean 147 pM), CV 1.8\% (mean $609 \mathrm{pM}$ ) and CV 2.4\% (mean 2,285 pM), in 25 separate runs in two months. The same controls were used for both assays (pooled serum in the lower, middle and higher range stored at $\left.-20^{\circ} \mathrm{C}\right)$. The Lumipulse method was compared to a LC-MS/MS method developed in the Erasmus Medical Centre (Waters Xevo-TQ-S, Waters Corporation, Inc., Milford, USA).

The LC-MS/MS method was calibrated using a commercially available reference standard (Aldosterone Cerillant No. A-096). Trueness of the method was assessed by external proficiency testing (SKML, The Netherlands). Proficiency was within limits set by the distributor for a reviewed period of one complete year both in terms of bias and imprecision. During this period, mean bias was $1.7 \%$ when compared to the LC-MS/MS consensus ( $n=9$ participants). For Lumipulse, no peers were available in any scheme due to the recent introduction of the assay.

The LC-MS/MS method was based on denaturation of the sample followed by Solid Phase Extraction (OASIS MAX $\mu$ Elution 96 well plate). The eluate was injected on the reversed phase UPLC column (Acquity UPLC BEH Phenyl column, $1.7 \mu \mathrm{M}, 2.1 \mathrm{~mm} \times 50 \mathrm{~mm}$ ) using a gradient of deionized water and methanol in $4 \mathrm{~min}$. Aldosterone was 
qualified and quantified at transitions 359.3 to 189.1 and 359.3 to $297.1 \mathrm{~m} / \mathrm{z}$, respectively. The cone voltage was set to $40 \mathrm{~V}$ and the collision energy to 18 and $14 \mathrm{~V}$, respectively. Imprecision of the LC-MS/MS method was CV 5.5\% (mean $138 \mathrm{pM}$ ), CV 5.8\% (mean $584 \mathrm{pM}$ ) and CV 5.3\% (mean 2,246 pM), measured over a period of one year in $\sim 70$ runs.

The Lumipulse assay was compared to LC-MS/MS in both routine ( $\mathrm{n}=39)$ and AVS ( $\mathrm{n}=133$ of 50 unique patients) with an aldosterone concentration up to $\sim 600$ and $\sim 300.000 \mathrm{pM}$ (LC-MS/MS), respectively. Samples that were not within the range of linearity (Lumipulse; $22-5,548 \mathrm{pM}$ and LC-MS/MS; 50-4,420 pM) were diluted. A dilution test was performed for the Lumipulse assay using Specimen Diluent 1, Fujirebio. The mean recovery found in $10 \mathrm{sam}-$ ples when diluted 200 times vs. 10 times was $108 \%$ and when diluted 10 times vs. undiluted 99\%. For the LC-MS/ MS assay the mean recovery found in five samples was $109 \%$ when diluted 200 times vs. 4 times and $107 \%$ in when diluted 4 times vs. undiluted in 10 samples using steroid free serum (Golden West, Temecula, CA) as diluent.

In the AVS sample set, the anatomical location was known in a subset $(\mathrm{n}=107)$. In addition to AVS samples, leftover routine samples were measured to compare the methods in a more common concentration range. Care was taken not to introduce systematic bias by randomizing measurements of anatomical locations over multiple runs.

Deming regression analysis was performed. The LC-MS/MS and Lumipulse G system assays showed strong correlations. No proportional or absolute bias was found between assays in leftover routine samples (Figure 1a). In the AVS measurements a proportional bias was found $(+6 \%, 95 \% \mathrm{CI}+3 \%$ to $+8 \%$ ) (Figure $1 \mathrm{~b})$. When split between anatomical locations, there was no bias for the peripheral samples $(n=41)$ or left adrenal samples $(n=30)$.
In the right adrenal samples $(\mathrm{n}=36)$ we found a proportional bias $(+6 \%, 95 \% \mathrm{CI}+2 \%$ to $+10 \%)$. Bias (\%) of the Lumipulse method relative to the LC-MS/MS method in routine measurements and AVS measurements per location is expressed using Bland-Altman plots (Figure 2).

The small bias found in samples from the right adrenal vein was not confounded by between run bias, as all samples were randomly measured in a two LC-MS/MS runs and in $>5$ runs on the Lumipulse system.

Since we have no information on the underlying pathology of the samples collected, it could be that our sample set was enriched with patients with either simply higher or aberrant output of the right adrenal of other substances. Given the average of the measured aldosterone concentrations (left $90.356 \mathrm{pM}$, right $60.132 \mathrm{pM}$ ) this is an unlikely explanation, which is further underscored by the fact that lateralization is more commonly seen on the left side [5]. However, we cannot exclude that high levels of aldosterone precursors or other steroids are selectively produced by the right adrenal.

Indeed, samples collected from the right and left adrenal vein contains extremely high steroid concentrations other than, but highly similar to, aldosterone which are known to be secreted in dissimilar amounts on the tumor and non-tumor side [4]. For example, in cases of PA, the ratio 18 -hydroxycorticosterone/aldosterone is 1.5 to 3 -fold on the tumor side, and five fold on the non-tumor side. In our case, the highest aldosterone concentration measured by mass spectrometry in any of the samples was $<3.5 \times 10^{5}$ $\mathrm{pM}$ which would result in an expected maximum concentration of 18-hydroxycorticosterone of $1.8 \times 10^{6} \mathrm{pM}$. Based on the manufacturers IFU, 18-hydroxycorticosterone has only very limited cross reactivity at this concentration level (18-hydroxycorticosterone $5 \times 10^{-4} \%$ at $2.7 \times 10^{6} \mathrm{pM}$ ), and
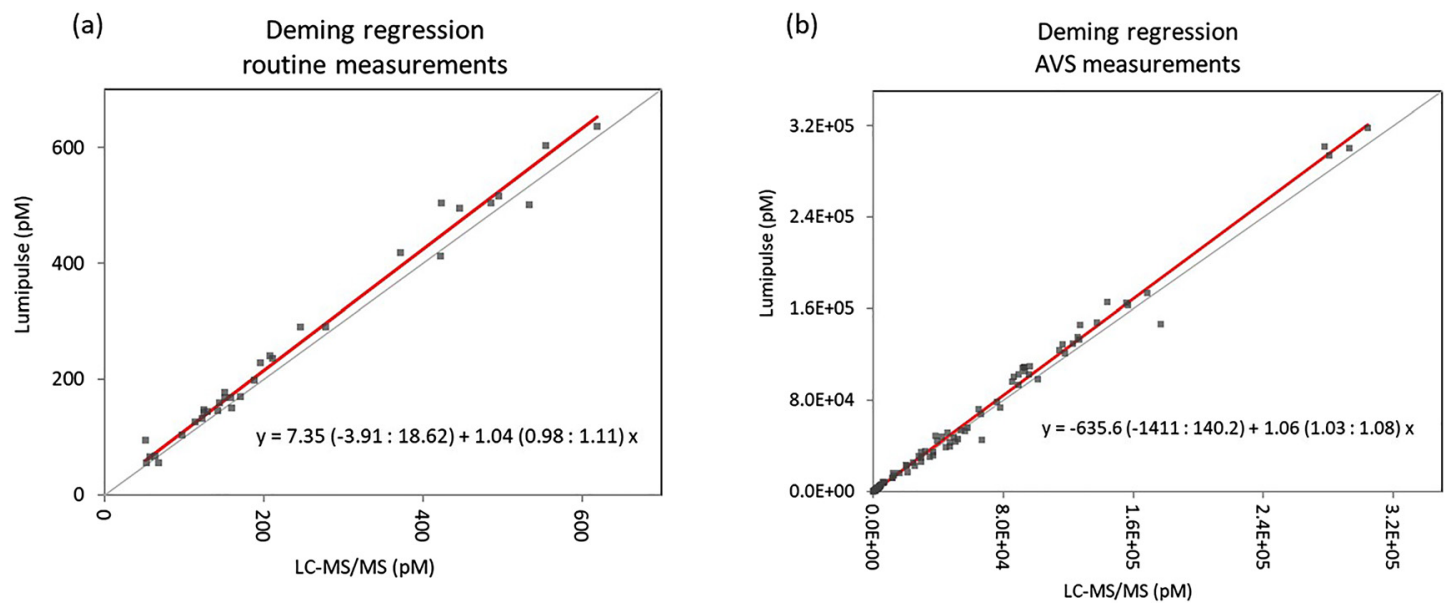

Figure 1: Aldosterone measured using LC-MS/MS (x-axis) vs. using the Lumipulse $\mathrm{G}$ system (y-axis). Aldosterone measured in left-over routine (a) and adrenal vein sampling samples (b). 

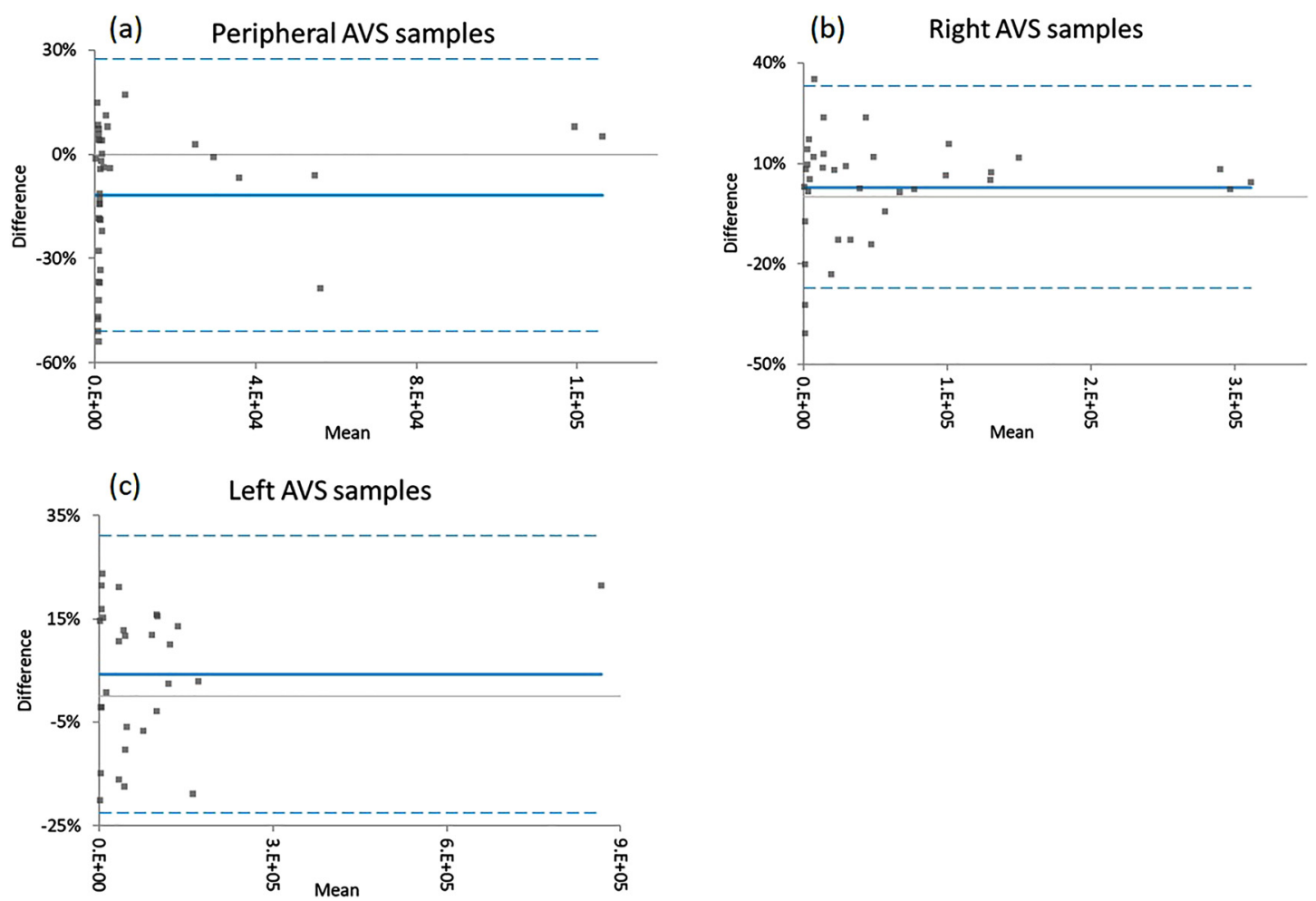

Figure 2: Bias per anatomical location of the Lumipulse assay relative to the LC-MS/MS assay.

Average between methods ( $x$-axis) vs. difference (y-axis).

would thus not explain the bias of $6 \%$. Unfortunately, therefore, we cannot elude the reason for bias at this moment.

Our results show that the Fujirebio Lumipulse $G$ System Aldosterone assay can be used for adrenal vein sampling in patients who suffer from primary hyperaldosteronism, although care must be taken for patients with marginal lateralization on the right side even though the $6 \%$ bias is marginal when compared to the conditions needed to be met for lateralization (lateralization index $>4$ at the dominant side compared to the nondominant side) [4].

Research funding: None declared.

Author contributions: All authors have accepted responsibility for the entire content of this manuscript and approved its submission.

Competing interests: Authors state no conflict of interest. Ethical approval: Not applicable.

\section{References}

1. Funder JW, Carey RM, Mantero F, Murad MH, Reincke M, Shibata H, et al. The management of primary aldosteronism: case detection, diagnosis, and treatment: an endocrine society clinical practice guideline. J Clin Endocrinol Metab 2016;101:1889-916.

2. Zennaro MC, Boulkroun S, Fernandes-Rosa FL. Pathogenesis and treatment of primary aldosteronism. Nat Rev Endocrinol 2020;16: 578-89.

3. Dekkers T, Prejbisz A, Kool LJS, Groenewoud HJMM, Velema M, Spiering W, et al. Adrenal vein sampling versus $\mathrm{CT}$ scan to determine treatment in primary aldosteronism: an outcome-based randomised diagnostic trial. Lancet Diabetes Endocrinol 2016;4: 739-46.

4. Rossi GP, Auchus RJ, Brown M, Lenders JWM, Naruse M, Plouin PF, et al. An expert consensus statement on use of adrenal vein sampling for the subtyping of primary aldosteronism. Hypertension 2014;63:151-60.

5. Miyoshi A, Wada N, Baba S, Obara S, Takahashi B, Usubuchi H, et al. Left-right differences in adrenal vein sampling for primary aldosteronism. Endocr J 2020;67:327-34. 